(C) 2004 International Press

Adv. Theor. Math. Phys. 8 (2004) 319-343

\title{
Transition from big crunch to big bang in brane cosmology
}

\author{
Claus Gerhardt \\ Institut für Angewandte Mathematik \\ Ruprecht-Karls-Universität \\ 69120 Heidelberg, Germany \\ gerhardt@math.uni-heidelberg.de
}

\begin{abstract}
We consider branes $N=I \times \mathcal{S}_{0}$, where $\mathcal{S}_{0}$ is an $n$-dimensional space form, not necessarily compact, in a Schwarzschild- $\operatorname{AdS}_{(n+2)}$ bulk $\mathcal{N}$. The branes have a big crunch singularity. If a brane is an ARW space, then, under certain conditions, there exists a smooth natural transition flow through the singularity to a reflected brane $\hat{N}$, which has a big bang singularity and which can be viewed as a brane in a reflected Schwarzschild-AdS ${ }_{(n+2)}$ bulk $\hat{\mathcal{N}}$. The joint branes $N \cup \hat{N}$ can thus be naturally embedded in $\mathbb{R}^{2} \times \mathcal{S}_{0}$, hence there exists a second possibility of defining a smooth transition from big crunch to big bang by requiring that $N \cup \hat{N}$ forms a $C^{\infty}$-hypersurface in $\mathbb{R}^{2} \times \mathcal{S}_{0}$. This last notion of a smooth transition also applies to branes that are not ARW spaces, allowing a wide range of possible equations of state.
\end{abstract}

\section{Introduction}

The problem of finding a smooth transition from a spacetime $N$ with a big crunch to a spacetime $\hat{N}$ with a big bang singularity has been the focus of some recent works in general relativity, see e.g., $[6,8]$ and the references therein. For abstract spacetimes, i.e., for spacetimes that are not embedded in a bulk space, it is even a non-trivial question how to define a smooth transition.

e-print archive: http://lanl.arXiv.org/abs/gr-qc/0404061 
In two recent papers $[2,4]$ we studied this problem for a special class of spacetimes, so-called ARW spaces, and used the inverse mean curvature flow to prove that, by reflecting the spaces, a smooth transition from big crunch to big bang is possible.

In this paper we look at branes in a Schwarzschild-AdS ${ }_{(n+2)}$ bulk $\mathcal{N}$, where the branes are assumed to lie in the black hole region, i.e., the radial coordinate is the time function. For those branes that are ARW spaces the transition results from [4] can be applied to conclude that a smooth transition flow from a brane $N$ to a properly reflected brane $\hat{N}$ exists. However, the assumption that the branes are ARW spaces reduces the number of possible branes drastically, cf. Theorem 0.4 and Theorem 0.5. Fortunately, in the case of embedded spacetimes, it is possible to define a transition through the singularity without using the inverse mean curvature flow.

Let $\mathcal{N}$ be a Schwarzschild-AdS ${ }_{(n+2)}$ bulk space with a black hole singularity in $r=0$. We assume that the radial coordinate $r$ is negative, $r<0$. Then, by switching the light cone and changing $r$ to $-r$ we obtain a reflected Schwarzschild-AdS ${ }_{(n+2)}$ bulk space $\hat{\mathcal{N}}$ with a white hole singularity in $r=0$. These two bulk spacetimes can be pasted together such that $\mathcal{N} \cup \hat{\mathcal{N}}$ is a smooth manifold, namely, $\mathbb{R}^{2} \times \mathcal{S}_{0}$, which has a metric singularity in $r=0$. In the black (white) hole region $r$ is the time function and it is smooth across the singularity.

Now, let us consider branes $N$ in the black hole region of $\mathcal{N}$. These branes need not to be ARW spaces, they are only supposed to satisfy the first of the five assumptions imposed for ARW spaces. We call those branes to be of class $(B)$, cf. Definition 0.1.

The relation between the geometry of the branes and physics is governed by the Israel junction condition.

We shall prove existence and transition through the singularity only for single branes, but this does include a two branes or multiple branes configuration-where then each brane has to be treated separately.

Moreover, in the equation of state

$$
p=\frac{\omega}{n} \rho
$$

variable $\omega$ are allowed

$$
\omega=\omega_{0}+\lambda, \quad \omega_{0}=\text { const },
$$

where $\lambda=\lambda(\log (-r))$ is defined in the bulk. 
Branes of class $(B)$ exist in the whole black hole region of $\mathcal{N}$, they stretch from $r=0$, the black hole singularity, to $r=r_{0}$, the event horizon.

The branes whose existence is proved in Theorem 3.1 automatically have a big crunch singularity in $r=0$, since this is the initial condition for the ordinary differential equation that has to be solved. The branes are given by an embedding

$$
y(\tau)=\left(r(\tau), t(\tau), x^{i}\right), \quad-a<\tau<0,
$$

with a big crunch singularity in $\tau=0$ such that $r(0)=0$.

From the modified Friedmann equation we shall deduce that the limit

$$
\lim _{\tau \rightarrow 0} t(\tau)=t_{0}
$$

exists and without loss of generality we shall suppose $t_{0}=0$.

We then shall define a brane $\hat{N} \subset \hat{\mathcal{N}}$ by reflection

$$
\hat{y}(\tau)=\left(-r(-\tau),-t(-\tau), x^{i}\right), \quad 0<\tau<a .
$$

The two branes $N, \hat{N}$ can be pasted together to yield at least a Lipschitz hypersurface in $\mathbb{R}^{2} \times \mathcal{S}_{0}$. If this hypersurface is of class $C^{\infty}$, then we shall speak of a smooth transition from big crunch to big bang.

To prove the smoothness we reparametrize $N \cup \hat{N}$ by using $r$ as a new parameter instead of $\tau$. The old brane $N$ is then expressed as

$$
y(r)=\left(r, t(r), x^{i}\right), \quad r<0,
$$

and the reflected $\hat{N}$ as

$$
\hat{y}(r)=\left(r,-t(-r), x^{i}\right), \quad r>0 .
$$

Hence, $N \cup \hat{N}$ is a smooth hypersurface, if $\frac{d t}{d r}$ is a smooth and even function in $r$ in a small neighbourhood of $r=0,-\epsilon<r<\epsilon$.

If $\frac{d t}{d r}$ is not an even function in $r$, then $N \cup \hat{N}$ will not be $C^{\infty}$ hypersurface. Examples can easily be constructed.

For branes, that are ARW spaces, the transition flow provides an alternate criterion for a smooth transition through the singularity. The reflected brane $\hat{N}$ that is used in this process is the same as in the above description of pasting together the two embeddings. 
A version of the modified Friedmann equation, equation (1.18), plays a central role in determining if the transition is smooth, namely, the quantity $f^{\prime} e^{\tilde{\gamma} f}$ has to be a smooth and even function in the variable $e^{\tilde{\gamma} f}$ in order that the transition flow is smooth, and a smooth and even function in the variable $r=-e^{f}$, if the joint embedding is to represent a smooth hypersurface in $\mathbb{R}^{2} \times \mathcal{S}_{0}$.

The metric in the bulk space $\mathcal{N}$ is given by

$$
d \tilde{s}^{2}=-\tilde{h}^{-1} d r^{2}+\tilde{h} d t^{2}+r^{2} \sigma_{i j} d x^{i} d x^{j},
$$

where $\left(\sigma_{i j}\right)$ is the metric of an $n$ - dimensional space form $\mathcal{S}_{0}$, the radial coordinate $r$ is assumed to be negative, $r<0$, and $\tilde{h}(r)$ is defined by

$$
\tilde{h}=m(-r)^{-(n-1)}+\frac{2}{n(n+1)} \Lambda r^{2}-\tilde{\kappa},
$$

where $m>0$ and $\Lambda \leq 0$ are constants, and $\tilde{\kappa}=-1,0,1$ is the curvature of $\mathcal{S}_{0}$. We note that we assume that there is a black hole region, i.e., if $\Lambda=0$, then we have to suppose $\tilde{\kappa}=1$.

We consider branes $N$ contained in the black hole region $\left\{r_{0}<r<0\right\}$. $N$ will be a globally hyperbolic spacetime $N=I \times \mathcal{S}_{0}$ with metric

$$
d \bar{s}^{2}=e^{2 f}\left(-\left(d x^{0}\right)^{2}+\sigma_{i j} d x^{i} d x^{j}\right)
$$

such that

$$
f=f\left(x^{0}\right)=\log \left(-r\left(x^{0}\right)\right)
$$

We may assume that the time variable $x^{0}=\tau$ maps $N$ on the interval $(-a, 0)$. In $\tau=0$ we have a big crunch singularity induced by the black hole.

The relation between geometry and physics is governed by the Israel junction conditions

$$
h_{\alpha \beta}-H \bar{g}_{\alpha \beta}=\kappa\left(T_{\alpha \beta}-\sigma \bar{g}_{\alpha \beta}\right),
$$

where $h_{\alpha \beta}$ is the second fundamental form of $N, H=\bar{g}^{\alpha \beta} h_{\alpha \beta}$ the mean curvature, $\kappa \neq 0$ a constant, $T_{\alpha \beta}$ the stress energy tensor of a perfect fluid with an equation of state

$$
p=\frac{\omega}{n} \rho,
$$

and $\sigma$ the tension of the brane.

One of the parameters used in the definition of $(n+1)$-dimensional ARW spaces is a positive constant $\tilde{\gamma}$, which is best expressed as

$$
\tilde{\gamma}=\frac{1}{2}(n+\tilde{\omega}-2) \text {. }
$$


If $N$ would satisfy the Einstein equation of a perfect fluid with an equation of state

$$
p=\frac{\tilde{\omega}}{n} \rho,
$$

then $\tilde{\gamma}$ would be defined with the help of $\tilde{\omega}$ in $(0.15)$, cf. [2, Section 9].

ARW spaces with compact $\mathcal{S}_{0}$ also have a future mass $\tilde{m}>0$, which is defined by

$$
\tilde{m}=\lim \int_{M} G_{\alpha \beta} \nu^{\alpha} \nu^{\beta} e^{\tilde{\omega} f},
$$

where $G_{\alpha \beta}$ is the Einstein tensor and where the limit is defined with respect to a sequence of spacelike hypersurfaces running into the future singularity, cf. [5].

For ARW spaces with non-compact $\mathcal{S}_{0}$ we simply call the limit

$$
\lim \left|f^{\prime}\right|^{2} e^{\tilde{\gamma} f}=\tilde{m}
$$

which exists by definition and is positive, mass.

The most general branes that we consider are branes of class $(B)$, they are supposed to satisfy only the first of the five conditions that are imposed on ARW spaces, cf. Definition 0.8.

Let us formulate this condition as

0.1 Definition. A globally hyperbolic spacetime $N, N=I \times \mathcal{S}_{0}, I=$ $(a, b)$, the metric of which satisfies (0.10), with $f=f\left(x^{0}\right)$, is said to be of class $(B)$, if there exist positive constants $\tilde{\gamma}$ and $\tilde{m}$ such that

$$
\lim _{\tau \rightarrow b}\left|f^{\prime}\right|^{2} e^{2 \tilde{\gamma} f}=\tilde{m}>0
$$

We also say that $N$ is of class $(B)$ with constant $\tilde{\gamma}$ and call $\tilde{m}$ the mass of $N$, though, even in the case of compact $\mathcal{S}_{0}$, the relation $(0.16)$ is defined only under special circumstances.

The time function in spacetimes of class $(B)$ has finite future range, cf. [2, Lemma 3.1], thus we may - and shall — assume that $b=0$ and $I=(-a, 0)$.

By considering branes of class $(B)$ instead of ARW spaces a larger range of equation of states is possible

$$
p=\frac{\omega}{n} \rho
$$

We shall also consider variable $\omega$. 
0.2 Lemma. Let $T_{\alpha \beta}$ be the divergence free stress energy tensor of a perfect fluid in $N$ with an equation of state

$$
p=\frac{\omega}{n} \rho,
$$

where $\omega=\omega_{0}+\lambda(f)$ and $\omega_{0}=$ const. Assume that $\lambda$ is smooth satisfying

$$
\lim _{t \rightarrow-\infty} \lambda(t)=0
$$

and let $\tilde{\mu}=\tilde{\mu}(t)$ be a primitive of $\lambda$ such that

$$
\lim _{t \rightarrow-\infty} \tilde{\mu}(t)=0
$$

Then $\rho$ satisfies the conservation law

$$
\rho=\rho_{0} e^{-\left(n+\omega_{0}\right) f-\tilde{\mu}},
$$

where $\rho_{0}$ is a constant.

A proof will be given in Section 1.

0.3 Remark. Since the branes, we shall consider, always satisfy the assumptions

$$
\lim _{\tau \rightarrow 0} f=-\infty, \quad-f^{\prime}>0
$$

it is possible to define $\mu=\mu(r), r=-e^{f}$, by

$$
\mu(r)=\tilde{\mu}(\log (-r)) .
$$

We also call $\mu$ a primitive of $\lambda$.

The main results of this paper can now be summarized in the following four theorems.

0.4 Theorem. Let $N$ be a brane contained in the black hole region of $\mathcal{N}$. Let $n \geq 3$ and assume that $\lambda=\lambda(t)$ satisfies

$$
\left|D^{m} \lambda(t)\right| \leq c_{m} \quad \forall m \in \mathbb{N} .
$$

(i) If $\sigma \geq 0$, then $\tilde{\gamma}=\frac{1}{2}(n-1)$ is the only possible value such that $N$ is an ARW space. 
(ii) On the other hand, if we set $\tilde{\gamma}=\frac{1}{2}(n-1)$, then $N$ is an ARW space if and only if the following conditions are satisfied

$$
\begin{gathered}
\omega_{0}=-\frac{n-1}{2} \quad \text { and } \quad\left\{\begin{array}{lll}
\sigma=0, & \text { if } & 3<n \in \mathbb{N}, \\
\sigma \in \mathbb{R}, & \text { if } & n=3,
\end{array}\right. \\
\quad\left|D_{t}^{m} \lambda\right| \leq c_{m} e^{(n-1) t} \quad \forall m \in \mathbb{N}
\end{gathered}
$$

and

$$
\lim _{t \rightarrow-\infty} \lambda(t) e^{-(n-1) t}
$$

exists, or

$$
\omega_{0} \leq-(n-1) \quad \text { and } \quad \sigma \in \mathbb{R}, \forall 3 \leq n \in \mathbb{N} .
$$

If the condition (0.27) holds, then the mass $\tilde{m}$ of $N$ is larger than $m$

$$
\tilde{m}=m+\frac{\kappa^{2}}{n^{2}} \rho_{0}^{2},
$$

where $\rho_{0}$ is an integration constant of $\rho e^{\left(n+\omega_{0}\right) f} e^{\tilde{\mu}}$. In the other cases we have $\tilde{m}=m$.

(iii) There exists a smooth transition flow from $N$ to a reflected brane $\hat{N}$, if the primitive $\mu$ can be viewed as a smooth and even function in $e^{\tilde{\gamma} f}$, and provided the following conditions are valid

$$
n=3, \quad \omega_{0}=-\frac{n-1}{2}, \quad \sigma \in \mathbb{R}
$$

or

$$
n>3, \quad \omega_{0}=-\frac{n-1}{2}, \quad \Lambda=\sigma=0, \quad \tilde{\kappa}=1,
$$

or

$$
n=3, \quad \omega_{0}=-m(n-1)+1,2 \leq m \in \mathbb{N}, \quad \sigma \in \mathbb{R},
$$

or

$$
n>3, \quad \omega_{0}=-m(n-1)+1,2 \leq m \in \mathbb{N}, \quad \frac{2}{n(n+1)} \Lambda=-\sigma^{2},
$$

where in case $\Lambda=\sigma=0$, we have to assume $\tilde{\kappa}=1$.

(iv) Since an $A R W$ brane is also a brane of class $(B)$, the smooth transition results from Theorem 0.7 , (i), are valid, if the primitive $\mu$ can be viewed as a smooth and even function in the variable $r=-e^{f}$.

(v) For the specified values of $\omega_{0}$ and $\sigma$ the branes do actually exist. 
0.5 Theorem. Assume that $\lambda$ vanishes in a neighbourhood of $-\infty$. Then a brane $N \subset \hat{\mathcal{N}}$ is an ARW space with $\tilde{\gamma} \neq \frac{1}{2}(n-1)$ if and only if $\tilde{\gamma}=n$, $\omega_{0}=1$, and

$$
\sigma=-\frac{m}{2 \rho_{0}}
$$

The mass $\tilde{m}$ of $N$ is then equal to

$$
\tilde{m}=\frac{\kappa^{2}}{n^{2}} \rho_{0}^{2} .
$$

(i) There exists a smooth transition flow, if

$$
\frac{2}{n(n+1)} \Lambda=-\sigma^{2} \text {. }
$$

(ii) Since $N$ is also a brane of class $(B)$, the smooth transition result from Theorem 0.7, (ii), is valid, i.e., the joint branes $N \cup \hat{N}$ form a $C^{\infty}$ hypersurface in $\mathbb{R}^{2} \times \mathcal{S}_{0}$, if $n \geq 3$ is odd, since $\mu$ can be viewed as a smooth and even function in $r$.

A brane with the specified values does actually exist.

0.6 Theorem. A brane $N \subset \mathcal{N}$ satisfying an equation of state with $\omega=\omega_{0}+\lambda$, where $\lambda$ satisfies the conditions of Lemma 0.2 , is of class $(B)$ with constant $\tilde{\gamma}>0$ if and only if

$$
\tilde{\gamma}=\frac{n-1}{2} \text { and } \omega_{0} \leq-\frac{n-1}{2},
$$

or

$$
\tilde{\gamma}=n+\omega_{0}-1 \quad \text { and } \quad \omega_{0}>-\frac{n-1}{2} .
$$

In both cases the tension $\sigma \in \mathbb{R}$ can be arbitrary. Branes with the specified values do actually exist.

0.7 Theorem. Let $N$ be a brane of class $(B)$ as described in the preceding theorem and assume that the corresponding function $\mu$ satisfies the conditions stated in (2.7) resp. (2.8), which more or less is tantamount to requiring that $\mu$ is smooth and even as a function of $r$. Then $N$ can be reflected to yield a brane $\hat{N} \subset \hat{\mathcal{N}}$. The joint branes $N \cup \hat{N}$ form a $C^{\infty}$. hypersurface in $\mathbb{R}^{2} \times \mathcal{S}_{0}$ provided the following conditions are valid

(i) If $\tilde{\gamma}=\frac{n-1}{2}$ and $\omega_{0} \leq-\frac{n-1}{2}$, then the relations

$$
\frac{n-1}{2} \text { odd, } \omega_{0} \text { odd, and } \sigma \in \mathbb{R},
$$

or

$$
n \text { odd }, \quad \omega_{0} \in \mathbb{Z}, \quad \text { and } \quad \sigma=0
$$


should hold.

(ii) If $\tilde{\gamma}=n+\omega_{0}-1$ and $\omega_{0}>-\frac{n-1}{2}$, then the relations

$$
n \text { odd, } \omega_{0} \text { odd, and } \sigma \in \mathbb{R},
$$

or

$$
n \text { odd }, \quad \omega_{0} \in \mathbb{Z}, \quad \text { and } \quad \sigma=0
$$

should be valid.

For the convenience of the reader we repeat the definition of ARW spaces, slightly modified to include the case of non-compact $\mathcal{S}_{0}$.

0.8 Definition. A globally hyperbolic spacetime $N, \operatorname{dim} N=n+1$, is said to be asymptotically Robertson-Walker (ARW) with respect to the future, if a future end of $N, N_{+}$, can be written as a product $N_{+}=[a, b) \times \mathcal{S}_{0}$, where $\mathcal{S}_{0}$ is a Riemannian space, and there exists a future directed time function $\tau=x^{0}$ such that the metric in $N_{+}$can be written as

$$
d \breve{s}^{2}=e^{2 \tilde{\psi}}\left\{-\left(d x^{0}\right)^{2}+\sigma_{i j}\left(x^{0}, x\right) d x^{i} d x^{j}\right\},
$$

where $\mathcal{S}_{0}$ corresponds to $x^{0}=a, \tilde{\psi}$ is of the form

$$
\tilde{\psi}\left(x^{0}, x\right)=f\left(x^{0}\right)+\psi\left(x^{0}, x\right)
$$

and we assume that there exists a positive constant $c_{0}$ and a smooth Riemannian metric $\bar{\sigma}_{i j}$ on $\mathcal{S}_{0}$ such that

$$
\lim _{\tau \rightarrow b} e^{\psi}=c_{0} \wedge \lim _{\tau \rightarrow b} \sigma_{i j}(\tau, x)=\bar{\sigma}_{i j}(x),
$$

and

$$
\lim _{\tau \rightarrow b} f(\tau)=-\infty
$$

Without loss of generality we shall assume $c_{0}=1$. Then $N$ is ARW with respect to the future, if the metric is close to the Robertson-Walker metric

$$
d \bar{s}^{2}=e^{2 f}\left\{-d x^{0^{2}}+\bar{\sigma}_{i j}(x) d x^{i} d x^{j}\right\}
$$

near the singularity $\tau=b$. By close we mean that the derivatives of arbitrary order with respect to space and time of the conformal metric $e^{-2 f} \breve{g}_{\alpha \beta}$ in (0.45) should converge to the corresponding derivatives of the conformal limit metric in (0.49), when $x^{0}$ tends to $b$. We emphasize that in our terminology 
Robertson-Walker metric does not necessarily imply that $\left(\bar{\sigma}_{i j}\right)$ is a metric of constant curvature, it is only the spatial metric of a warped product.

We assume, furthermore, that $f$ satisfies the following five conditions

$$
-f^{\prime}>0
$$

there exists $\tilde{\omega} \in \mathbb{R}$ such that

$$
n+\tilde{\omega}-2>0 \wedge \lim _{\tau \rightarrow b}\left|f^{\prime}\right|^{2} e^{(n+\tilde{\omega}-2) f}=\tilde{m}>0 .
$$

Set $\tilde{\gamma}=\frac{1}{2}(n+\tilde{\omega}-2)$, then there exists the limit

$$
\lim _{\tau \rightarrow b}\left(f^{\prime \prime}+\tilde{\gamma}\left|f^{\prime}\right|^{2}\right)
$$

and

$$
\left|D_{\tau}^{m}\left(f^{\prime \prime}+\tilde{\gamma}\left|f^{\prime}\right|^{2}\right)\right| \leq c_{m}\left|f^{\prime}\right|^{m} \quad \forall m \geq 1
$$

as well as

$$
\left|D_{\tau}^{m} f\right| \leq c_{m}\left|f^{\prime}\right|^{m} \quad \forall m \geq 1
$$

If $\mathcal{S}_{0}$ is compact, then we call $N$ a normalized ARW spacetime, if

$$
\int_{\mathcal{S}_{0}} \sqrt{\operatorname{det} \bar{\sigma}_{i j}}=\left|S^{n}\right|
$$

0.9 Remark. The special branes we consider are always RobertsonWalker spaces, i.e., in order to prove that they are also ARW spaces we only have to show that $f$ satisfies the five conditions stated above.

\section{The modified Friedmann equation}

The Israel junction condition (0.12) is equivalent to

$$
h_{\alpha \beta}=\kappa\left(T_{\alpha \beta}-\frac{1}{n} T \bar{g}_{\alpha \beta}+\frac{\sigma}{n} \bar{g}_{\alpha \beta}\right),
$$

where $T=T_{\alpha}^{\alpha}$.

Assuming the stress energy tensor to be that of a perfect fluid

$$
T_{0}^{0}=-\rho, \quad T_{i}^{\alpha}=p \delta_{i}^{\alpha},
$$

satisfying an equation of state

$$
p=\frac{\omega}{n} \rho,
$$


we finally obtain

$$
h_{i j}=\frac{\kappa}{n}(\rho+\sigma) \bar{g}_{i j}
$$

for the spatial components of the second fundamental form.

Let us label the coordinates in $\mathcal{N}$ as $\left(y^{a}\right)=\left(y^{r}, y^{t}, y^{i}\right) \equiv\left(r, t, x^{i}\right)$. Then we consider embeddings of the form

$$
y=y\left(\tau, x^{i}\right)=\left(r(\tau), t(\tau), x^{i}\right)
$$

$x^{0}=\tau$ should be the time function on the brane which is chosen such that the induced metric can be written as

$$
d \bar{s}^{2}=r^{2}\left(-\left(d x^{0}\right)^{2}+\sigma_{i j} d x^{i} d x^{j}\right) .
$$

We also assume that $\dot{r}>0$. Notice that $r<0$, so that $\left(x^{\alpha}\right)$ is a future oriented coordinate system on the brane. If we set $f=\log (-r)$, then the induced metric has the form as indicated in (0.10).

Let us point out that this choice of $\tau$ implies the relation

$$
r^{2}=\tilde{h}^{-1} \dot{r}^{2}-\tilde{h}\left|t^{\prime}\right|^{2}
$$

since

$$
\bar{g}_{00}=\langle\dot{y}, \dot{y}\rangle,
$$

where we use a dot or a prime to indicate differentiation with respect to $\tau$ unless otherwise specified.

Since the time function in ARW spaces or in spaces of class $(B)$ has a finite future range, cf. [2, Lemma 3.1], we assume without loss of generality that the embedding is defined in $I \times \mathcal{S}_{0}$ with $I=(-a, 0)$.

The only non-trivial tangent vector of $N$ is

$$
y^{\prime}=\left(r^{\prime}, t^{\prime}, 0 \ldots, 0\right),
$$

and hence a covariant normal $\left(\nu_{a}\right)$ of $N$ is given by

$$
\lambda\left(-t^{\prime}, r^{\prime}, 0, \ldots, 0\right),
$$

where $\lambda$ is a normalization factor, and the contravariant normal vector is given by

$$
\left(\nu^{a}\right)=-r^{-1}\left(\tilde{h} \frac{d t}{d \tau}, \tilde{h}^{-1} \dot{r}, 0, \ldots, 0\right)
$$

in view of (1.7). 
The normal vector $\nu$ of the brane is spacelike, i.e., the Gauß formula reads

$$
y_{\alpha \beta}=-h_{\alpha \beta} \nu
$$

we refer to [1, Section 2] for our conventions.

We also emphasize that we have neither specified the sign nor the actual value of $\kappa$, i.e., it is irrelevant which normal we use in the Gauß formula.

To determine $h_{i j}$ we use

$$
y_{i j}^{t}=y_{, i j}^{t}+\tilde{\Gamma}_{b c}^{t} y_{i}^{b} y_{j}^{c}-\bar{\Gamma}_{i j}^{\gamma} y_{\gamma}^{t}=-r^{-1} \dot{r} \frac{d t}{d \tau} \sigma_{i j},
$$

and we conclude

$$
h_{i j}=-\tilde{h} \frac{d t}{d \tau} \sigma_{i j}
$$

in view of (1.12), (1.13) and the assumption $\dot{r} \neq 0$, and from (1.4) we further deduce

$$
-\tilde{h} r^{-2} \frac{d t}{d \tau}=\frac{\kappa}{n}(\rho+\sigma),
$$

or, by taking (1.7) into account,

$$
\left|f^{\prime}\right|^{2}-\tilde{h}=\frac{\kappa^{2}}{n^{2}}(\rho+\sigma)^{2} e^{2 f} .
$$

This is the modified Friedmann equation.

Branes that are $A R W$ spaces

Let us first consider branes that are ARW spaces.

Since the bulk is an Einstein space, the left-hand side of (0.12) is divergence free, as can be easily deduced with the help of the Codazzi equation, i.e., $T_{\alpha \beta}$ is also divergence free, and hence $\rho$ satisfies the conservation law

$$
\rho e^{\left(n+\omega_{0}\right) f} e^{\tilde{\mu}}=\mathrm{const}=\rho_{0},
$$

cf. Lemma 0.2; a proof will be given later.

In order to find out under which conditions the brane is an ARW space, we distinguish between the cases $\sigma \geq 0$ and $\sigma<0$. The latter choice violates the approximation of the classical Friedmann equation for small $\rho$.

1.1 Lemma. Let $\sigma \geq 0$, then $\tilde{\gamma}=\frac{1}{2}(n-1)$ is the only possible value such that $N$ can be an ARW space. 
Proof. From (1.16) and (1.17) we derive

$$
\begin{aligned}
\left|f^{\prime}\right|^{2} e^{2 \tilde{\gamma} f}= & m e^{(2 \tilde{\gamma}-(n-1)) f}+\frac{2}{n(n+1)} \Lambda e^{2(\tilde{\gamma}+1) f}-\tilde{\kappa} e^{2 \tilde{\gamma} f} \\
+ & \frac{\kappa^{2}}{n^{2}}\left(\rho_{0}^{2} e^{2\left(\tilde{\gamma}+1-\left(n+\omega_{0}\right)\right) f} e^{-2 \tilde{\mu}}\right. \\
& \left.+2 \sigma \rho_{0} e^{\left(2 \tilde{\gamma}+2-\left(n+\omega_{0}\right)\right) f} e^{-\tilde{\mu}}+\sigma^{2} e^{2(\tilde{\gamma}+1) f}\right) .
\end{aligned}
$$

Differentiating both sides and dividing by $2 f^{\prime}$ yields

$$
\begin{aligned}
& \left(f^{\prime \prime}+\tilde{\gamma}\left|f^{\prime}\right|^{2}\right) e^{2 \tilde{\gamma} f}= \\
& m\left(\tilde{\gamma}-\frac{(n-1)}{2}\right) e^{(2 \tilde{\gamma}-(n-1)) f}+\frac{2(\tilde{\gamma}+1)}{n(n+1)} \Lambda e^{2(\tilde{\gamma}+1) f}-\tilde{\gamma} \tilde{\kappa} e^{2 \tilde{\gamma} f} \\
& +\frac{\kappa^{2}}{n^{2}}\left(\rho_{0}^{2}\left(\tilde{\gamma}+1-\left(n+\omega_{0}\right)\right) e^{2\left(\tilde{\gamma}+1-\left(n+\omega_{0}\right)\right) f} e^{-2 \tilde{\mu}}\right. \\
& -\lambda \rho_{0}^{2} e^{2\left(\tilde{\gamma}+1-\left(n+\omega_{0}\right)\right) f} e^{-2 \tilde{\mu}} \\
& +\sigma \rho_{0}\left(2 \tilde{\gamma}+2-\left(n+\omega_{0}\right)\right) e^{\left(2 \tilde{\gamma}+2-\left(n+\omega_{0}\right)\right) f} e^{-\tilde{\mu}} \\
& -\lambda \sigma \rho_{0} e^{\left(2 \tilde{\gamma}+2-\left(n+\omega_{0}\right)\right) f} e^{-\tilde{\mu}} \\
& \left.+\sigma^{2}(\tilde{\gamma}+1) e^{2(\tilde{\gamma}+1) f}\right) .
\end{aligned}
$$

If $N$ is an ARW space, then the left-hand side of (1.18) has to converge to a positive constant, if $\tau$ goes to zero, and $f^{\prime \prime}+\tilde{\gamma}\left|f^{\prime}\right|^{2}$ has to converge to a constant.

Thus we deduce that all exponents of $e^{f}$ on the right-hand side of (1.18) have to be non-negative. Dividing now equation (1.19) by $e^{2 \tilde{\gamma} f}$, and using the fact that the terms involving $\lambda$ can be neglected, since $\lambda$ vanishes sufficiently fast near $-\infty$, we see that the coefficients of all powers of $e^{f}$, which have the common factor $\frac{\kappa^{2}}{n^{2}}$, are non-negative, hence we must have $\tilde{\gamma}=\frac{1}{2}(n-1)$, for otherwise we get a contradiction.

1.2 Lemma. Let $\tilde{\gamma}=\frac{1}{2}(n-1), \sigma \in \mathbb{R}$ and assume that (0.26) is valid. Then $N$ is an ARW space if and only if the following conditions are satisfied

$$
\omega_{0}=-\frac{n-1}{2} \quad \text { and } \quad\left\{\begin{array}{lll}
\sigma=0, & \text { if } & 3<n \in \mathbb{N}, \\
\sigma \in \mathbb{R}, & \text { if } & n=3,
\end{array}\right.
$$

and the relations $(0.28)$ and $(0.29)$ hold, or

$$
\omega_{0} \leq-(n-1) \quad \text { and } \quad \sigma \in \mathbb{R}, \forall 3 \leq n \in \mathbb{N} .
$$


Proof. Let $n \geq 3$ and $\sigma \in \mathbb{R}$ be arbitrary. If the left-hand side of (1.18) converges, then the exponents of the terms with the common factor $\frac{\kappa^{2}}{n^{2}}$ have to be non-negative, since the exponents of the terms with the factor $\rho_{0}$ can't be both negative and equal, so that they might cancel each other. Hence there must hold

$$
\omega_{0} \leq-\frac{n-1}{2}
$$

Moreover, after dividing (1.19) by $e^{2 \tilde{\gamma} f}$ we see that either $\omega_{0}=-\frac{n-1}{2}$ or

$$
n+\omega_{0} \leq 1
$$

i.e.,

$$
\omega_{0} \leq-(n-1)
$$

In case $\omega_{0}=-\frac{n-1}{2}$, we deduce from (1.19) that either $\sigma=0$ or

$$
0 \leq 2-n+\frac{n-1}{2}=-\frac{n-3}{2},
$$

i.e., $n=3$ must be valid, if $\sigma \neq 0$.

If these necessary conditions are satisfied, then we can express $f^{\prime \prime}+\tilde{\gamma}\left|f^{\prime}\right|^{2}$ in the form

$$
\begin{aligned}
f^{\prime \prime}+\tilde{\gamma}\left|f^{\prime}\right|^{2} & =\frac{1}{n} \Lambda e^{2 f}-\tilde{\gamma} \tilde{\kappa} \\
+\frac{\kappa^{2}}{n^{2}}( & -\lambda \rho_{0}^{2} e^{-(n-1) f} e^{-2 \tilde{\mu}}+2 \sigma \rho_{0} e^{-\tilde{\mu}} \\
& \left.-\lambda \sigma \rho_{0} e^{-\tilde{\mu}}+\sigma^{2}(\tilde{\gamma}+1) e^{2 f}\right)
\end{aligned}
$$

in case $\omega_{0}=-\frac{n-1}{2}$, where we note that $\sigma=0$, if $n>3$, and $\tilde{\gamma}=1$, if $n=3$, and in the form

$$
\begin{aligned}
f^{\prime \prime}+\tilde{\gamma}\left|f^{\prime}\right|^{2} & =\frac{1}{n} \Lambda e^{2 f}-\tilde{\gamma} \tilde{\kappa} \\
+\frac{\kappa^{2}}{n^{2}}( & c_{1} e^{2 \epsilon_{1} f} e^{-2 \tilde{\mu}}-\lambda c_{2} e^{2 \epsilon_{1} f} e^{-2 \tilde{\mu}}+c_{3} e^{\left(\epsilon_{1}+1\right) f} e^{-\tilde{\mu}} \\
& \left.\quad-\lambda c_{4} e^{\left(\epsilon_{1}+1\right) f} e^{-\tilde{\mu}}+c_{5} e^{2 f}\right)
\end{aligned}
$$

with constants $c_{i}, \epsilon_{1}$, such that $\epsilon_{1} \geq 0$, if $\omega_{0} \leq-(n-1)$.

Thus the remaining conditions for $f$ in the definition of ARW spaces are automatically satisfied, in view of the conditions (0.26), (0.28), and (0.29).

On the other hand, it is immediately clear that the conditions in the lemma are also sufficient provided we have a solution of equation (1.16) such that

$$
\lim _{\tau \rightarrow 0} f=-\infty \quad \text { and } \quad f^{\prime}<0
$$


The existence of such a solution will be shown in Theorem 3.1. $\frac{n-1}{2}$.

Next let us examine the possibility that $N$ is an ARW space with $\tilde{\gamma} \neq$

1.3 Lemma. Let $\tilde{\gamma} \neq \frac{n-1}{2}$, and suppose that $\lambda$ vanishes in a neighbourhood of $-\infty$. Then $N$ is an ARW space with constant $\tilde{\gamma}$ if and only if $\tilde{\gamma}=n$, $\omega=1$, and $\sigma<0$ is fine tuned to

$$
\sigma=-\frac{m}{2 \rho_{0}}
$$

where $\rho_{0}$ is the integration constant in (1.17).

Proof. Let $N$ be an ARW space with $\tilde{\gamma} \neq \frac{n-1}{2}$, then we conclude from (1.18) that all exponents of $e^{f}$ had to be non-negative or

$$
2-\left(n+\omega_{0}\right)=-(n-1)
$$

i.e., $\omega_{0}=1$, and $\sigma$ had to be fine tuned as indicated in (1.29). If all exponents were non-negative, then we would use (1.19) to deduce the same result as in (1.30) with the corresponding value for $\sigma$.

Hence, in any case we must have $\omega_{0}=1$ and $\sigma$ as in (1.29). Inserting these values in (1.18) we conclude

$$
0=2 \tilde{\gamma}+2-2(n+1)
$$

i.e., $\tilde{\gamma}=n$.

The conditions in the lemma are therefore necessary. Suppose they are satisfied, then we deduce

$$
\lim \left|f^{\prime}\right|^{2} e^{2 \tilde{\gamma} f}=\frac{\kappa^{2}}{n^{2}} \rho_{0}^{2}
$$

and

$$
f^{\prime \prime}+\tilde{\gamma}\left|f^{\prime}\right|^{2}=\frac{2}{n} \Lambda e^{2 f}-\tilde{\gamma} \tilde{\kappa}+\frac{\kappa^{2}}{n^{2}} \sigma^{2}(n+1) e^{2 f},
$$

if $f$ is close to $-\infty$, from which we immediately infer that $N$ is an ARW space.

For the existence result we refer to Theorem 3.1. 
1.4 Remark. (i) If in Lemma 1.2 resp. Lemma $1.3 \omega_{0}$ is equal to the isolated values $\omega_{0}=-\frac{n-1}{2}$ resp. $\omega_{0}=1$, then the future mass of $N$ is different from the mass $m$ of $\mathcal{N}$, namely, in case $\omega_{0}=-\frac{n-1}{2}$ and $\tilde{\gamma}=\frac{n-1}{2}$ we get

$$
\tilde{m}=m+\frac{\kappa^{2}}{n^{2}} \rho_{0}^{2}>m,
$$

and in case $\omega_{0}=1$ and $\tilde{\gamma}=n$

$$
\tilde{m}=\frac{\kappa^{2}}{n^{2}} \rho_{0}^{2}
$$

(ii) In the case $\tilde{\gamma}=\frac{n-1}{2}$, the value of $\tilde{\gamma}$ is equal to the value that one would get assuming the Einstein equations were valid in $N$, where the stress energy tensor would be that of a perfect fluid with an equation of state

$$
p=\frac{\tilde{\omega}}{n} \rho
$$

such that $\tilde{\omega}=1$, since then $N$ would be an ARW space satisfying (0.14), cf. [2, Section 9].

Furthermore, the classical Friedmann equation has the form

$$
\left|f^{\prime}\right|^{2}=-\tilde{\kappa}+\frac{\kappa}{n} \rho e^{2 f}=-\tilde{\kappa}+\frac{\kappa}{n} \rho_{0} e^{(2-(n+\tilde{\omega})) f} .
$$

Thus, by identifying the leading powers of $e^{f}$ on the right-hand side of (1.16) and (1.37), we see that in case $\tilde{\omega}=1$ there should hold

$$
-(n+1)=-2\left(n+\omega_{0}\right),
$$

i.e.,

$$
\omega_{0}=-\frac{n-1}{2}
$$

Therefore, if we want to interpret the modified Friedmann equation in analogy to the classical Friedmann equation, then we have to choose $\omega_{0}=$ $-\frac{n-1}{2}$, if the relation (0.14) serves as a guiding principle.

Let us conclude these considerations on branes that are ARW spaces with the following lemma

1.5 Lemma. The branes $N$ which are ARW spaces satisfy the timelike convergence condition near the singularity. 
Proof. We apply the relation connecting the Ricci tensors of two conformal metrics. Let

$$
\left(\nu^{\alpha}\right)=\left(1, u^{i}\right) \quad \text { and } \quad v=1-\sigma_{i j} u^{i} u^{j} \equiv 1-|D u|^{2}>0
$$

then

$$
\begin{aligned}
\bar{R}_{\alpha \beta} \nu^{\alpha} \nu^{\beta} & =R_{i j} u^{i} u^{j}-(n-1)\left(f^{\prime \prime}-\left|f^{\prime}\right|^{2}\right)+v^{2}\left(-f^{\prime \prime}-(n-1)\left|f^{\prime}\right|^{2}\right) \\
& \geq(n-1) \tilde{\kappa}|D u|^{2}-(n-2) f^{\prime \prime}+(n-1)\left|f^{\prime}\right|^{2}|D u|^{2},
\end{aligned}
$$

hence the result.

Branes of class $(B)$

Next we shall examine branes that are of class $(B)$. Let us start with a proof of Lemma 0.2

Proof of Lemma 0.2. The equation $T_{0 ; \gamma}^{\gamma}=0$ implies

$$
0=-\dot{\rho}-(n+\omega) f^{\prime} \rho .
$$

If $\rho$ would vanish in a point, then it would vanish identically, in view of Gronwall's lemma. Hence, we may assume that $\rho>0$, so that

$$
(\log \rho)^{\prime}=-\left(n+\omega_{0}\right) f^{\prime}-\lambda f^{\prime},
$$

from which the conservation law (0.16) follows immediately.

In the following two lemmata we shall assume that $T_{\alpha \beta}$ satisfies an equation of state with $\omega=\omega_{0}+\lambda$, such that $\lambda, \tilde{\mu}$ and $\mu$ satisfy the relations (0.21), (0.22) and (0.25).

1.6 Lemma. Let $\tilde{\gamma}=\frac{n-1}{2}$ and $\omega=\omega_{0}+\lambda$. Then the brane $N$ is of class (B) with constant $\tilde{\gamma}$ if and only if

$$
\omega_{0} \leq-\frac{n-1}{2} .
$$

Proof. The left hand-side of equation (1.18) converges to a positive constant if and only if $\omega_{0}$ satisfies (1.44). 
1.7 Lemma. Let $\tilde{\gamma} \neq \frac{n-1}{2}, \rho>0$, and $\omega=\omega_{0}+\lambda$. Then the brane $N$ is of class $(B)$ with constant $\tilde{\gamma}$ if and only if

$$
\tilde{\gamma}=n+\omega_{0}-1
$$

and

$$
\omega_{0}>-\frac{n-1}{2}
$$

Proof. (i) Let $N$ be of class $(B)$ with constant $\tilde{\gamma} \neq \frac{n-1}{2}$, then

$$
n+\omega_{0}>0
$$

must be valid, for otherwise we get a contradiction. Therefore

$$
2\left(\tilde{\gamma}+1-\left(n+\omega_{0}\right)\right)
$$

is the smallest exponent on the right-hand side of (1.18) and has thus to vanish, i.e.,

$$
\tilde{\gamma}=n+\omega_{0}-1
$$

The other exponents have to be non-negative, i.e.,

$$
2 \tilde{\gamma}>n-1
$$

and

$$
2 \tilde{\gamma} \geq n+\omega_{0} \quad \text { or } \quad \sigma=0
$$

The inequality (1.50) is equivalent to (1.46) which in turn implies (1.51).

(ii) The conditions (1.45) and (1.46) are sufficient, since then

$$
2 \tilde{\gamma}>n-1
$$

and

$$
\tilde{m}=\frac{\kappa^{2}}{n^{2}} \rho_{0}^{2}
$$

Similarly as before branes of class $(B)$ do actually exist, cf. Theorem 3.1. 


\section{Transition from big crunch to big bang}

\section{Branes that are $A R W$ spaces}

For a brane $N$ that is an ARW space, the results of [4] can be applied. Define a reflected spacetime $\hat{N}$ by switching the lightcone and changing the time function to $\hat{x}^{0}=-x^{0}$, then $N$ and $\hat{N}$ can be pasted together at the singularity $\{0\} \times \mathcal{S}_{0}$ yielding a smooth manifold with a metric singularity which is a big crunch, when viewed from $N$, and a big bang, when viewed from $\hat{N}$. Moreover, there exists a natural transition flow of class $C^{3}$ across the singularity which is defined by rescaling an appropriate inverse mean curvature flow.

This transition flow is of class $C^{\infty}$, if the quantity $f^{\prime} e^{\tilde{\gamma} f}$, or equivalently, $\left|f^{\prime}\right|^{2} e^{2 \tilde{\gamma} f}$ can be viewed as a smooth and even function in the variable $e^{\tilde{\gamma} f}$, cf. [4, the remarks before Theorem 2.1].

For the branes considered in Theorem 0.4 and Theorem $0.5\left|f^{\prime}\right|^{2} e^{2 \tilde{\gamma} f}$ is a smooth and even function in $e^{\tilde{\gamma} f}$.

In the following we shall see that the reflected spacetime can be viewed as a brane in the Schwarzschild-AdS ${ }_{(n+2)}$ space $\hat{\mathcal{N}}$ which is a reflection of $\mathcal{N}$. $\hat{\mathcal{N}}$ is obtained by switching the lightcone in $\mathcal{N}$ and by changing the radial coordinate $r$ to $-r$. The singularity in $r=0$ is then a white hole singularity.

Moreover, ARW branes are also branes of class $(B)$, i.e., the transition results for those branes, which we shall prove next, also apply.

Branes of class (B)

The brane $N$ is given by

$$
y(\tau)=\left(r(\tau), t(\tau), x^{i}\right) .
$$

Since the coordinates $\left(x^{i}\right)$ do not change, let us write $y(\tau)=(r(\tau), t(\tau))$.

We shall see in a moment that $t(\tau)$ converges, if $\tau$ tends to zero, hence, let us set this limit equal to zero.

Now we define the reflection $\hat{N}$ of $N$ by

$$
\hat{y}(\tau)=(-r(-\tau),-t(-\tau))
$$


for $\tau>0$. The result is a brane in $\hat{\mathcal{N}}$ which can be pasted continuously to $N$.

The embeddings of $N$ resp. $\hat{N}$ in $\mathcal{N}$ resp. $\hat{\mathcal{N}}$ are also embeddings in $\mathbb{R}^{2} \times \mathcal{S}_{0}$, endowed with the Riemannian product metric, and now it makes sense to ask, if the joint hypersurfaces $N \cup \hat{N}$ form a smooth hypersurface in $\mathbb{R}^{2} \times \mathcal{S}_{0}$.

The smoothness of $N \cup \hat{N}$ in $\mathbb{R}^{2} \times \mathcal{S}_{0}$ we interpret as a smooth transition from big crunch to big bang.

To prove the smoothness we have to parameterize $y=y(\tau)$ with respect to $r$. This is possible, since

$$
\frac{d r}{d \tau}=-f^{\prime} e^{f}>0
$$

Then we have $y(r)=(r, t(r))$ and we need to examine

$$
\frac{d t}{d r}=\frac{d t}{d \tau} \frac{d \tau}{d r}=-\frac{d t}{d \tau} \frac{1}{f^{\prime} e^{f}} .
$$

Define

$$
\begin{aligned}
\vartheta(r) & =\tilde{h}(r) e^{(n-1) f} \\
& =m+\frac{2}{n(n+1)} \Lambda e^{(n+1) f}-\tilde{\kappa} e^{(n-1) f},
\end{aligned}
$$

then we obtain from (1.15) and (1.17)

$$
\begin{aligned}
\vartheta \frac{d t}{d r} & =\frac{\kappa}{n}\left(\sigma+\rho_{0} e^{-\left(n+\omega_{0}\right) f} e^{-\tilde{\mu}}\right) \frac{e^{n f}}{f^{\prime}} \\
& =\frac{\kappa}{n}\left(\sigma e^{(n+\tilde{\gamma}) f}+\rho_{0} e^{\left(\tilde{\gamma}-\omega_{0}\right) f} e^{-\tilde{\mu}}\right) \frac{1}{f^{\prime} e^{\tilde{\gamma} f}} .
\end{aligned}
$$

This immediately shows that in all cases $\left|\frac{d t}{d r}\right|$ is uniformly bounded, hence $\lim _{r \rightarrow 0} t(r)$ exists.

The joint embedding $N \cup \hat{N}$ in $\mathbb{R}^{2} \times \mathcal{S}_{0}$ will be of class $C^{\infty}$, if $\frac{d t}{d r}$ can be viewed as a smooth and even function in $r=-e^{f}$.

We shall assume that the function $\mu=\mu(r)$ in (0.25) is either even and smooth in $\left(-r_{0}, r_{0}\right)$

$$
\mu \in C^{\infty}\left(\left(-r_{0}, r_{0}\right)\right) \text { is even }
$$

or that $\mu \in C^{\infty}\left(\left(-r_{0}, 0\right]\right)$ such that

$$
\left|D^{m} \mu(0)\right|=0 \quad \forall m \in \mathbb{N} .
$$


In the latter case we can extend $\mu$ as an even and smooth function to $r>0$ by setting

$$
\mu(r)=\mu(-r) \quad \text { for } \quad r>0
$$

Then we can prove

2.1 Theorem. Let $N$ be a brane of class $(B)$ satisfying an equation of state with $\omega=\omega_{0}+\lambda$, so that $\lambda$ vanishes at the singularity. The corresponding function $\mu$ should satisfy the conditions (2.7) or (2.8). Then $N$ can be reflected as described above to yield a brane $\hat{N} \subset \hat{\mathcal{N}}$. The joint branes $N \cup \hat{N}$ form a $C^{\infty}$ - hypersurface in $\mathbb{R}^{2} \times \mathcal{S}_{0}$ provided the following conditions are valid

(i) If $\tilde{\gamma}=\frac{n-1}{2}$ and $\omega_{0} \leq-\frac{n-1}{2}$, then the relations

$$
\frac{n-1}{2} \text { odd, } \omega_{0} \text { odd, } \quad \text { and } \quad \sigma \in \mathbb{R},
$$

or

$$
n \text { odd, } \omega_{0} \in \mathbb{Z}, \quad \text { and } \quad \sigma=0
$$

should hold.

(ii) If $\tilde{\gamma}=n+\omega_{0}-1$ and $\omega_{0}>-\frac{n-1}{2}$, then the relations

$$
n \text { odd, } \omega_{0} \text { odd, and } \sigma \in \mathbb{R} \text {, }
$$

or

$$
n \text { odd, } \quad \omega_{0} \in \mathbb{Z} . \quad \text { and } \quad \sigma=0
$$

should be valid.

Proof. "(i)" Let $\tilde{\gamma}=\frac{n-1}{2}$ and $\omega_{0} \leq-\frac{n-1}{2}$. We have to show that $\frac{d t}{d r}$ is a smooth and even function in $r$. From equation (2.6) we deduce

$$
\frac{d t}{d r}=-\frac{\kappa}{n}\left(\sigma(-r)^{(n+\tilde{\gamma}) f}+\rho_{0} e^{\left(\tilde{\gamma}-\omega_{0}\right) f} e^{-\mu}\right) \vartheta^{-1} \varphi^{-1},
$$

where

$$
\begin{aligned}
\varphi^{2}=\left|f^{\prime}\right|^{2} e^{2 \tilde{\gamma} f}= & m e^{(2 \tilde{\gamma}-(n-1)) f}+\frac{2}{n(n+1)} \Lambda e^{2(\tilde{\gamma}+1) f}-\tilde{\kappa} e^{2 \tilde{\gamma} f} \\
+ & \frac{\kappa^{2}}{n^{2}}\left(\rho_{0}^{2} e^{2\left(\tilde{\gamma}+1-\left(n+\omega_{0}\right)\right) f} e^{-2 \mu}\right. \\
& \left.+2 \sigma \rho_{0} e^{\left(2 \tilde{\gamma}+2-\left(n+\omega_{0}\right)\right) f} e^{-\mu}+\sigma^{2} e^{2(\tilde{\gamma}+1) f}\right) .
\end{aligned}
$$

The right-hand side of (2.15) has to be an even and smooth function in $r=-e^{f}$. By assumption $\mu$ is already an even and smooth function or can be 
extended to such a function, hence we have to guarantee that the exponents of $e^{f}$ are all even.

A thorough examination of the exponents reveals that this is the case, if the conditions $(2.10)$ or $(2.11)$ are fulfilled. Notice that

$$
\frac{n-1}{2} \text { odd } \Longrightarrow n \text { odd } \wedge \frac{3 n-1}{2} \text { even }
$$

\section{„(ii)“ Identical proof.}

\section{Existence of the branes}

We want to prove that for the specified values of $\omega_{0}$ and corresponding $\tilde{\gamma}$ embedded branes $N$ in the black hole region of $\mathcal{N}$ satisfying the Israel junction condition do exist.

Evidently it will be sufficient to solve the modified Friedmann equation (1.16) on an interval $I=(-a, 0]$ such that, if we set $r=-e^{f}$, then

$$
\lim _{\tau \rightarrow 0} r(\tau)=0, \quad \lim _{\tau \rightarrow-a} r(\tau)=r_{0}
$$

where $r_{0}$ is the (negative) black hole radius, i.e., $\left\{r=r_{0}\right\}$ equals the horizon.

We shall assume the most general equation of state that we considered in the previous sections, i.e., $\omega$ should be of the form $\omega=\omega_{0}+\lambda(f)$, cf. Lemma 0.2 , and the corresponding primitive $\mu=\mu(r)$ should be smooth in $r_{0}<r<0$.

Let us look at equation (1.18). Setting $\varphi=e^{\tilde{\gamma} f}$ we deduce that it can be rewritten as

$$
\tilde{\gamma}^{-2} \dot{\varphi}^{2}=F(\varphi)
$$

where $F=F(t)$ is defined on an interval $J=\left[0 \leq t<t_{0}\right)$ and $t_{0}$ is given by

$$
t_{0}=\left(-r_{0}\right)^{\tilde{\gamma}}
$$

$F$ is continuous in $J$ and smooth in $\stackrel{\circ}{J}$. Furthermore, it satisfies

$$
F>0 \text { and } F(0)=\tilde{m}>0 .
$$


If we succeed in solving the equation

$$
\tilde{\gamma}^{-1} \dot{\varphi}=-\sqrt{F(\varphi)}
$$

with initial value $\varphi(0)=0$ on an interval $I=(-a, 0]$ such that $\varphi \in C^{0}(I) \cap$ $C^{\infty}(\stackrel{\circ}{I})$ and such that the relations $(3.1)$ are valid for $r=-\varphi^{-1}$, then the modified Friedmann equation is solved by $f=-\tilde{\gamma}^{-1} \log \varphi$ and $f$ satisfies

$$
\lim _{\tau \rightarrow 0} f=-\infty, \quad-f^{\prime}>0 .
$$

To solve (3.5) let us make a variable transformation $\tau \rightarrow-\tau$, so that we have to solve

$$
\tilde{\gamma}^{-1} \dot{\varphi}=\sqrt{F(\varphi)}
$$

with initial value $\varphi(0)=0$ on an interval $I=[0, a)$.

3.1 Theorem. The equation (3.7) with initial value $\varphi(0)=0$ has a solution $\varphi \in C^{1}(I) \cap C^{\infty}(\stackrel{\circ}{I})$, where $I=[0, a)$ is an interval such that the relations (3.1) are satisfied by $r=-\varphi^{\tilde{\gamma}^{-1}}$ with obvious modifications resulting from the variable transformation $\tau \rightarrow-\tau$.

Proof. Let $0<t^{\prime}<t_{0}$ be arbitrary and define $J^{\prime}=\left[0, t^{\prime}\right]$. Then there are positive constants $c_{1}, c_{2}$ such that

$$
c_{1}^{2} \leq F(t) \leq c_{2}^{2} \quad \forall t \in J^{\prime} .
$$

Let $\epsilon>0$ be small, then the differential equation (3.7) with initial value $\varphi_{\epsilon}(0)=\epsilon$ has a smooth, positive solution $\varphi_{\epsilon}$ in $0 \leq \tau<\tau_{\epsilon}$, where $\tau_{\epsilon}$ is determined by the requirement

$$
\varphi_{\epsilon}(\tau) \leq t^{\prime} \quad \forall 0 \leq \tau<\tau_{\epsilon} .
$$

In view of the estimates (3.8) we immediately conclude that

$$
\tilde{\gamma} c_{1} \tau \leq \varphi_{\epsilon}(\tau) \leq \epsilon+\tilde{\gamma} c_{2} \tau \quad \forall 0 \leq \tau<\tau_{\epsilon},
$$

hence, if we choose the maximal $\tau_{\epsilon}$ possible, then there exists $\tau_{0}>0$ such that

$$
\tau_{\epsilon} \geq \tau_{0}>0 \quad \forall \epsilon
$$

Now, using well-known a priori estimates, we deduce that for any interval $I^{\prime} \Subset\left(0, \tau_{0}\right)$ we have

$$
\left|\varphi_{\epsilon}\right|_{m, I^{\prime}} \leq c_{m}\left(I^{\prime}\right) \quad \forall m \in \mathbb{N}
$$


independently of $\epsilon$. Moreover, since $\varphi_{\epsilon}$ is uniformly Lipschitz continuous in $\left[0, \tau_{0}\right]$, we infer that

$$
\lim _{\epsilon \rightarrow 0} \varphi_{\epsilon}=\varphi
$$

exists such that $\varphi \in C^{1}\left(\left[0, \tau_{0}\right]\right) \cap C^{\infty}\left(\left(0, \tau_{0}\right)\right)$ and $\varphi$ solves $(3.7)$ with initial value $\varphi(0)=0$.

$\varphi$ can be defined on a maximal interval interval $\left[0, a^{*}\right)$, where $a^{*}$ is determined by

$$
\lim _{\tau \rightarrow a^{*}} \varphi(\tau)=\infty \quad \text { or } \quad \lim _{\tau \rightarrow a^{*}} F(\varphi)=0
$$

Obviously, there exists $0<a \leq a^{*}$ such that

$$
\lim _{\tau \rightarrow a} \varphi(\tau)=\left(-r_{0}\right)^{\tilde{\gamma}}
$$

Hence the proof of the theorem is completed.

3.2 Remark. If $a<a^{*}$, then we cannot conclude that we have defined branes extending beyond the horizon, since our embedding deteriorates when the horizon is approached, as a look at the equations (1.15) and (1.16) reveals. Since $\tilde{h}$ vanishes on the horizon, either $\frac{d t}{d \tau}$ becomes unbounded or $(\rho+\sigma)$ would tend to zero, in which case $F(\varphi)$ would vanish, i.e., if $a<a^{*}$, then necessarily

$$
\lim _{\tau \rightarrow-a}\left|\frac{d t}{d \tau}\right|=\infty
$$

has to be valid. An extension of the brane past the horizon will require a different embedding or at least a different coordinate system in the bulk. Notice that

$$
\bar{g}_{00}=\langle\dot{y}, \dot{y}\rangle=-r^{2} \neq 0,
$$

so that it is more or less a coordinate singularity.

Acknowledgement. This work has been supported by the Deutsche Forschungsgemeinschaft.

\section{References}

[1] Claus Gerhardt, Hypersurfaces of prescribed curvature in Lorentzian manifolds, Indiana Univ. Math. J. 49 (2000), 1125-1153, pdf file.

[2] _ The inverse mean curvature flow in ARW spaces - transition from big crunch to big bang, 2004, 39 pages, math.DG/0403485. 
[3] - The inverse mean curvature flow in cosmological spacetimes, 2004, 24 pages, math.DG/0403097.

[4] - The inverse mean curvature flow in Robertson-Walker spaces and its application to cosmology, 2004, 9 pages, gr-qc/0404112.

[5] - The mass of a Lorentzian manifold, 2004, 14 pages, math.DG/0403002.

[6] Justin Khoury, A briefing on the ekpyrotic/cyclic universe, 2004, 8 pages, astro-ph/0401579.

[7] David Langlois, Brane cosmology: an introduction, 2002, 32 pages, hepth/0209261.

[8] Neil Turok and Paul J. Steinhardt, Beyond inflation: A cyclic universe scenario, 2004, 27 pages, hep-th/0403020. 